\title{
Effects on EEG of Low (1Hz) and High (15Hz) Frequency Repetitive Transcranial Magnetic Stimulation of the Visual Cortex: A Study in the Anesthetized Cat
}

\author{
N. Espinosa ${ }^{\#, 1}$, C. de Labra ${ }^{\#, 1}$, C. Rivadulla ${ }^{1}$, J. Mariño ${ }^{1}$, K.L. Grieve ${ }^{1,2}$ and J. Cudeiro*,1 \\ ${ }^{I}$ Neuroscience and Motor Control Group (NEUROcom), Department of Medicine, Universidad de A Coruña, Campus \\ de Oza, 15006 A Coruña, Spain \\ ${ }^{2}$ Faculty of Life Sciences, The University of Manchester, M60 1QD,UK
}

\begin{abstract}
Here we confirm our earlier findings that showed 1Hz rTMS over the primary visual cortex of the anesthetized cat, known to cause inhibition of the cortex, induces an increase in power in the slow, delta band of the EEG. We also demonstrate that these inhibitory effects of $1 \mathrm{~Hz}$ rTMS may be measured as changes of spatiotemporal receptive field parameters at the single cell level. We extend these observations to show that higher frequency stimulation of the cortex at $15 \mathrm{~Hz}$ has the opposite effect of decreasing delta activity, which was also accompanied by significant increases in theta, alpha and beta bands. This highly reproducible EEG change may be useful as a simple marker to predict inhibitory or excitatory rTMS effects known to be dependent upon stimulation frequency.
\end{abstract}

\section{INTRODUCTION}

Repetitive transcranial magnetic stimulation (rTMS) is a novel research tool with applications in neurology and psychiatry. It has been used to treat brain diseases or improve functional deficits [1,2]. In addition, based upon early evidence showing that trains of stimuli delivered to the motor cortex could produce either increased or decreased corticospinal excitability [3-5], rTMS has become an excellent procedure for testing and modulating neuronal excitability. Low frequencies of stimulation (in the range of $1 \mathrm{~Hz}$ or below) produce inhibitory changes in excitability [6] whilst higher rates (above $5 \mathrm{~Hz}$ ) appear to produce increasing excitability (with concomitant risk of epileptic episodes) [3,7,8].

We have recently used rTMS [9] as a tool to inhibit the activity of the visual cortex while recording single cells in the lateral geniculate nucleus (dLGN) of the anesthetized cat. While we showed that $1 \mathrm{~Hz}$ stimulation appeared to be inhibitory, and caused cortical suppression, a much stronger and longer inhibition was obtained if trains of high frequency were applied. This paradoxical result was explained by the long inter-train interval we selected, which effectively changed a high frequency stimulation paradigm into low frequency [9]. We have previously demonstrated that the low frequency stimulation effectively increased the low frequency delta band activity of the EEG in our anesthetized cat model [10], a finding reinforced by others [11]. In the present work we investigated and compared low $(1 \mathrm{~Hz})$ and high $(15 \mathrm{~Hz})$ TMS frequency stimulation and reveal that these have opposing effects on the EEG. Further, we report single cell data supporting the suppressive nature of the low frequency stimulation. Our results suggest that a simple skull mounted EEG could be used to determine if a TMS paradigm will result in inhibition or excitation.

\footnotetext{
*Address correspondence to this author at the Neuroscience and Motor Control Group (NEUROcom), Department of Medicine, Universidad de A Coruña, Campus de Oza, 15006 A Coruña, Spain; E-mail: jcud@udc.es

${ }^{\#} \mathrm{~N}$. Espinosa and C. de Labra contributed equally to this work.
}

\section{MATERIALS AND METHODS}

Four adult cats of either sex were prepared using standard procedures used in our laboratory for experiments involving extracellular recordings along the visual pathway $[9,12]$. Animals were anaesthetised with halothane in nitrous oxide $(70 \%)$ and oxygen $(30 \%)$ (halothane $-1.5-2 \%$ for surgery and $0.1-0.5 \%$ for maintenance). Animals were initially anesthetized intramuscularly with a ketamine $(15-20 \mathrm{mg} / \mathrm{kg}$ ) and xylazine $(1 \mathrm{mg} / \mathrm{kg})$ mixture. To prevent eye movements, animals were paralysed with gallamine triethiodide (loading dose of $40 \mathrm{mg}$, maintenance $10 \mathrm{mg} / \mathrm{kg} / \mathrm{hr}$ IV infusion). End-tidal $\mathrm{CO}_{2}$ levels, ECG waveform, intersystolic interval and EEG were monitored continuously throughout the experiment. The rate and depth of artificial ventilation were adjusted to maintain end-tidal $\mathrm{CO}_{2}$ at 3.8-4.2\%; the level of halothane was chosen to achieve a state of light anaesthesia. Once a stable state was reached, any variation in the monitored parameters commensurate with a change in the depth of anaesthesia was compensated for by alterations in the level of halothane (effects on EEG as a result of the experimental paradigm would not have been considered to change anaesthetic status unless accompanied by changes in other parameters; this was not seen). Wound margins were treated with lidocaine hydrochloride administered subcutaneously. Ear bars were coated with lidocaine gel. The eyes were treated with atropine methonitrate and phenylephrine hydrochloride, protected with zero power contact lenses and brought to focus on a semi-opaque tangent screen $57 \mathrm{~cm}$ distant using ancillary lenses. Visual stimuli were viewed monocularly through $3 \mathrm{~mm}$ artificial pupils. To further reduce possible eye movement artefacts, rigid posts were fixed to the sclera and attached to the stereotaxic frame. The procedures conformed to the Spanish Physiology Society, the International Council for Laboratory Animal Science and the European Union (statute no. 86/809) and The Physiological Society (UK) regulations. 


\section{Receptive Fields Mapping and Data Acquisition}

In three experiments (aimed primarily to study the role of corticofugal feedback in controlling thalamic activity) [9], single unit electrophysiological recordings were also obtained from neurons located in the striate visual cortex of the cat (V1) using tungsten electrodes. Electrodes were either advanced during the experiment at an oblique angle (avoiding the stimulating coil) or cemented in place (tip located approximately in layer 6) prior to positioning of the TMS coil. Spatiotemporal receptive fields of the cells were mapped using the technique described by Cai et al. (1997) [13], which is a one-dimensional version of the reverse correlation technique $[14,15]$. For all quantitative measures, visual stimuli were presented on a computer monitor (refresh rate $128 \mathrm{~Hz}$ ) $57 \mathrm{~cm}$ from the eye, centered on the center of the receptive field under study. Briefly, the visual stimulus was (Lohmann Research Equipment, Germany) a pseudorandom sequence of bright and dark bars that were briefly flashed (40ms) at 20 locations across the RF. Stimulus parameters were adjusted to cover the entire length and width
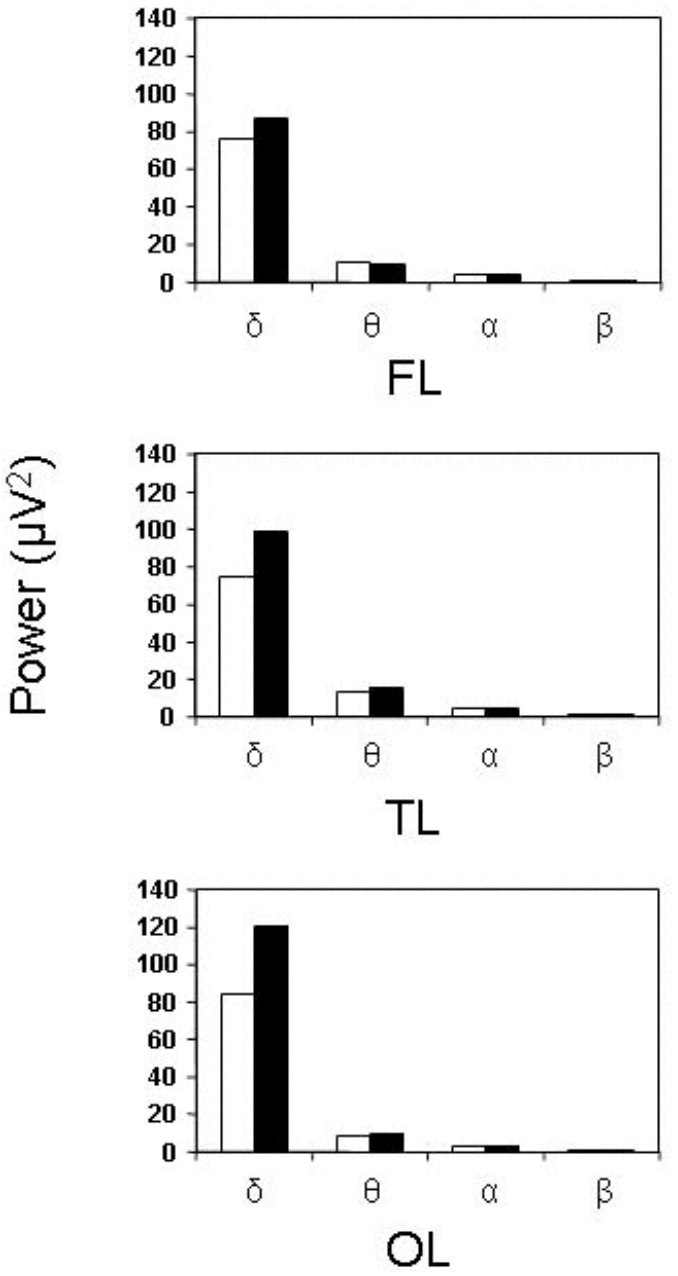

of the RF and were presented at the preferred orientation for each cell. The bar stimuli were typically $12^{\circ}$ in length and $0.2-0.5^{\circ}$ in width, and were usually presented for $40 \mathrm{~ms}$. These parameters were varied somewhat from cell to cell, according to the spatial and temporal resolution of the RF. In general, the stimuli were chosen to be as narrow as possible in both space and time but still elicit a measurable response from the neuron. For a stimulus patch having 20 locations, one stimulus sequence consisted of 40 different bar stimuli (20 locations x 2 contrast polarities, dark and bright). Typically, the stimulus sequence was repeated 100-200 times in order to obtain enough spikes (on the order of several thousand) for a smooth RF profile. Separate histograms were built up over time from the responses to bright and dark bars. Receptive fields maps were obtained before (control RF), immediately after the TMS and after a recovery period (15 $\min )$.

\section{EEG Recordings}

After the skin of the head was retracted, surface electrodes for EEG recording were placed bilaterally on the skull
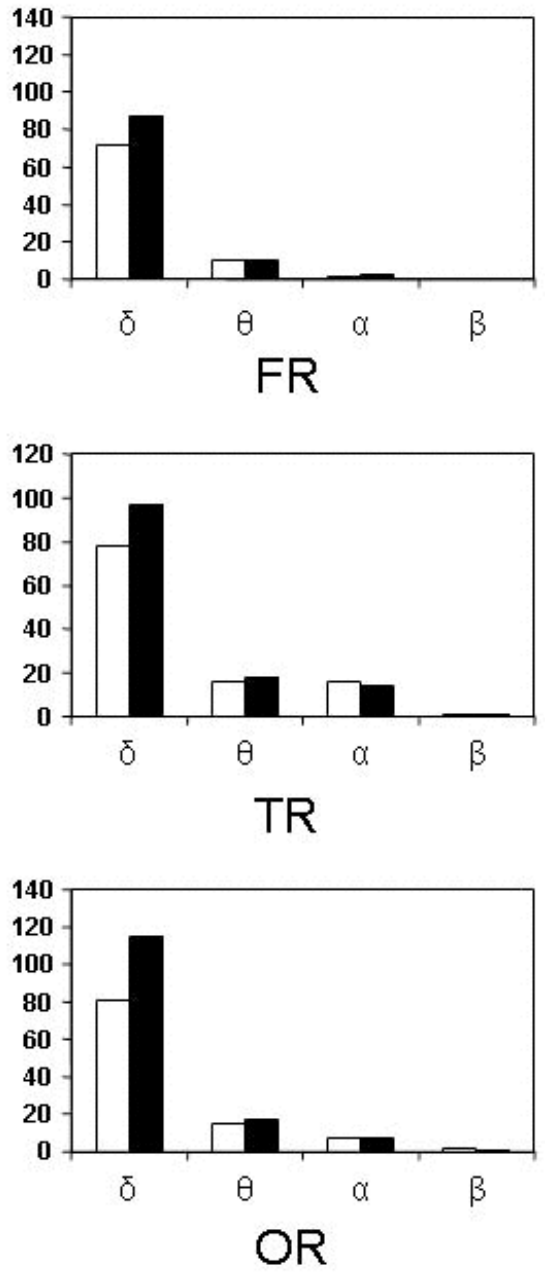

\section{before $\square$ after $\square$ rTMS@1 $1 \mathrm{~Hz}$}

Fig. (1). Histograms representation of the power spectral components of the EEG, recorded at 6 sites across the cat skull. Each histogram shows the power for the control condition (open bars) and that immediately following rTMS application over the visual cortex (solid bars). These are the results from a single application of rTMS at $1 \mathrm{~Hz}$, as outlined in Methods 
at frontal, temporal and occipital areas. EEG bandwidth was $0.1-100 \mathrm{~Hz}$, and the signal was sampled at $349 \mathrm{~Hz}$. The power spectrum of recorded cortical activity was calculated for a number of periods (10-15) of 5 seconds, before and after rTMS. Two different rTMS protocols were used:

1. Low frequency rTMS (50\% of maximal output) was delivered at $1 \mathrm{~Hz}$ for 3 minutes after a 20-30 min control period of EEG recording. Stimulation was followed by an inter-stimulus interval of 1 minute, after which rTMS was applied again for another 3 minutes. Then we recorded continuously for a period of 5-10 minutes. This experimental block was repeated a number of times (5-6) during each experiment and between blocks we waited at least 30 minutes.

2. High frequency rTMS $(15 \mathrm{~Hz}, 50 \%$ of maximal strength). After a similar 20-30 min control period of EEG recording, six trains of $1 \mathrm{sec}$ (inter-train interval, $5 \mathrm{sec})$ at $15 \mathrm{~Hz}$ were applied. After that we recorded continuously for a period of 5-10 minutes. This sequence was repeated a number of times (5-6) during each experiment and between blocks we waited at least 30 minutes.

Both protocols were tested in the same animal, separated by an interval of at least 2 hours. Power spectra of EEG recordings from the stimulated area (occipital pole, centre of TMS) and from the non-stimulated area (temporal and frontal cortex of both hemispheres) were analysed. Four frequency bands were analysed with regard to spectral power: delta band $(0.5-3 \mathrm{~Hz})$, theta band $(4-7 \mathrm{~Hz})$ alpha band $(8-13$ $\mathrm{Hz}$ ), beta band (14-32 Hz). Mean \pm SEM. were calculated for the spectral power of the EEG $\left(\mathrm{V}^{2}\right)$. An ANOVA test was used and a $P$ value $<0.05$ was considered significant.

rTMS was carried out with a MagStim Rapid system (The MagStim Company Ltd, UK) equipped with two boosters and applied to the occipital cortex via a figure-of-eight coil $(2 \times 25 \mathrm{~mm})$. The midpoint of the coil was centred over the inter-hemispheric cranial suture at the level of Brodmann's area 17 in direct contact with the bone. The coil was fixed by a mechanical arm at an angle of about $60^{\circ}$ (wings located -laterally) with the handle pointing up and backward.
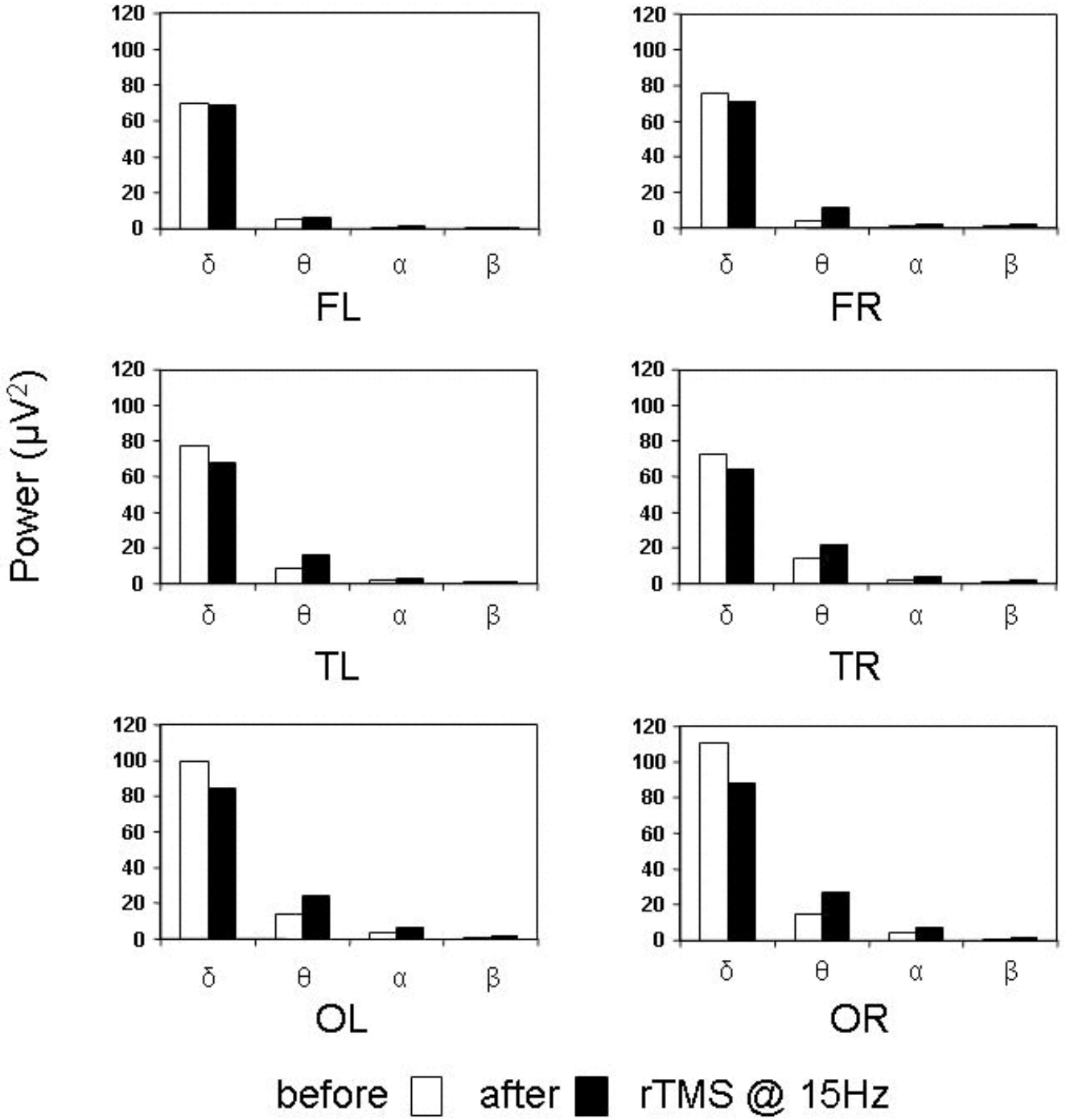

Fig. (2). Histograms representation of the power spectral components of the EEG. Recordings from the same animal as figure1, here using TMS at 15Hz. Again, each histogram shows the power for the control condition (open bars) and that immediately following TMS application over the visual cortex (solid bars). These are the results from a single application of TMS, here at $15 \mathrm{~Hz}$, as outlined in Methods 


\section{RESULTS}

\section{Effects of Low Frequency rTMS (1Hz) on EEG}

Fig. (1) shows the effects of $1 \mathrm{~Hz}$ rTMS application on the EEG power spectrum. White bars illustrate the spectral power of the EEG recorded for the $60 \mathrm{sec}$ period immediately prior to $3 \mathrm{~min}$ of rTMS at $1 \mathrm{~Hz}$. Solid bars represent the EEG power values obtained in the first $5 \mathrm{~s}$ after rTMS. The most obvious finding was a clear increment of power in the low $\delta$ band In this example, the other bands were unaffected. The observed modifications in the FFT power pattern recovered gradually, and after 5 minutes the values returned to the control situation (not shown).

\section{Effects of High Frequency rTMS (15Hz) on EEG}

Fig. (2) illustrates the spectral power of the EEG before and after the "15hz train" rTMS. The observed effect was essentially the opposite of the one shown in the previous figure; delta power was significantly decreased, and, here, power increased in the theta range. Other values were not significantly altered.

The single examples shown above indicate opposing effects of the 2 TMS regimes, particularly on delta band power. To analyze this in more detail, we have compiled average values for all the animals tested, presented in Fig. (3). The cartoon on the left represents the arrangement of the electrodes, the central spatial point of the TMS is marked by *. A clear tendency appeared: following $1 \mathrm{~Hz}$ TMS stimulation (top table), the very low frequencies in the spectrum (delta band) increased, theta frequency was unchanged and medium (alpha) and high frequencies (beta) tended to diminish. Asterisks mark those changes which are statistically significant. The poor spatial resolution of EEG is apparent, with the effects seen essentially equally over all electrodes (but see below and discussion).

Crucially, the effect of $15 \mathrm{hHz}$ TMs (lower table) was basically opposite - a significant decrease in the delta band and concomitant increase in theta, alpha and beta. Here, however, this increased power was not seen at the level of the temporal electrodes.

\section{Effects of rTMS (1Hz) on the Spatiotemporal Responses of V1 Neurons}

Fig. (4A) shows a spatiotemporal receptive field profile ( $S T$ plot) for a V1 neuron from an adult cat obtained before, immediately after and 15 minutes after rTMs at $1 \mathrm{~Hz}$. Spatial position is plotted along the vertical axis and time is plotted on the horizontal axis.

There is a clear reduction in the response of the cell after the application of rTMS together with a change in the spatiotemporal profile. We have focused our attention on the observed changes in the temporal receptive field structure. To study that we found the spatial location at which the spatiotemporal profile has its largest positive value. A temporal response profile is then obtained by slicing through the spatiotemporal profile at this position (for further details see Cai et al. 1997). This is shown in Fig. (4B). The blue line illustrates the profile of the cell in the control situation, which reaches it maximum at $40.8 \mathrm{~ms}$ (peak time). The red trace shows the response of the cell after rTMS. The decrement in cell firing is immediately obvious, and is accompanied by a delay $(3.1 \mathrm{~ms})$ in reaching peak value. Further, the duration of the response, as measured at half height, decreases from 27.3 to $19 \mathrm{~ms}$.

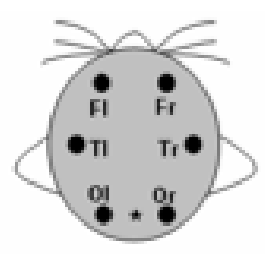

rTMS@1 $1 \mathrm{~Hz}$

\begin{tabular}{|c|l|l|l|l|}
\hline Electrodes & Delta & Theta & Alpha & Beta \\
\hline Fl control & $74 \pm 8.9$ & $13 \pm 3.8$ & $9 \pm 2$ & $2 \pm 0.2$ \\
TMS & $78 \pm 10$ & $11 \pm 2.7$ & $9 \pm 1$ & $1 \pm 0.1^{*}$ \\
\hline Fr control & $71 \pm 7.8$ & $12 \pm 4$ & $10 \pm 3$ & $1.7 \pm 0.2$ \\
TMS & $80 \pm 9.1^{*}$ & $11 \pm 3.1$ & $7 \pm 2^{*}$ & $1.1 \pm 0.1^{*}$ \\
\hline Tl control & $80 \pm 7.7$ & $15 \pm 3.9$ & $7 \pm 1.7$ & $2.3 \pm 0.3$ \\
TMS & $90 \pm 7.3^{*}$ & $16 \pm 4$ & $6 \pm 1.3$ & $1 \pm 0.2^{*}$ \\
\hline Tr control & $74 \pm 9$ & $17 \pm 4.2$ & $11 \pm 3$ & $2 \pm 0.2$ \\
TMS & $83 \pm 7.9^{*}$ & $19 \pm 4.2$ & $7 \pm 1.2^{*}$ & $2 \pm 0.3$ \\
\hline OI control & $82 \pm 8.2$ & $13 \pm 4.2$ & $9 \pm 2$ & $2.3 \pm 0.2$ \\
TMS & $94 \pm 8.8^{*}$ & $13 \pm 4.3$ & $5 \pm 0.8^{*}$ & $1 \pm 0.1^{*}$ \\
\hline Or control & $79 \pm 8$ & $12 \pm 4.1$ & $12 \pm 2.8$ & $2 \pm 0.2$ \\
TMS & $90 \pm 8.9^{*}$ & $13 \pm 4$ & $8 \pm 1.9^{*}$ & $0.7 \pm 0.1^{*}$ \\
\hline
\end{tabular}

rTMS@15Hz

\begin{tabular}{|c|l|l|l|l|}
\hline Electrodes & Delta & Theta & Alpha & Beta \\
\hline Fl control & $79 \pm 7$ & $10 \pm 3$ & $8 \pm 2 /$ & $1.9 \pm 0.1$ \\
TMS & $61 \pm 6.3^{*}$ & $12 \pm 3$ & $6 \pm 3.8^{*}$ & $2.1 \pm 0.1$ \\
\hline Fr contl & $81 \pm 7$ & $12 \pm 2.9$ & $9 \pm 2.1$ & $1 \pm 0.08$ \\
TMS & $62 \pm 6.1^{*}$ & $20 \pm 3.3^{*}$ & $16 \pm 3.7^{*}$ & $2 \pm 0.2^{*}$ \\
\hline Tl control & $86 \pm 8$ & $16 \pm 3.8$ & $6 \pm 0.9$ & $2 \pm 0.2$ \\
TMS & $69 \pm 6.3^{*}$ & $17 \pm 3.7$ & $7 \pm 1$ & $2 \pm 0.1$ \\
\hline Tr control & $83 \pm 8.1$ & $18 \pm 4$ & $8 \pm 1.3$ & $2 \pm 0.15$ \\
TMS & $67 \pm 6.5^{*}$ & $17 \pm 3.8$ & $10 \pm 2.2$ & $2 \pm 0.1$ \\
\hline OI control & $83 \pm 9$ & $11 \pm 3.2$ & $7 \pm 1.9$ & $1.8 \pm 0.1$ \\
TMS & $61 \pm 7^{*}$ & $19 \pm 4^{*}$ & $13 \pm 3.7^{*}$ & $2.8 \pm 0.3^{*}$ \\
\hline Or control & $80 \pm 8.1$ & $12 \pm 3.3$ & $9 \pm 2$ & $2 \pm 0.2$ \\
TMS & $62 \pm 7^{*}$ & $20 \pm 3.9^{*}$ & $16 \pm 4^{*}$ & $3.2 \pm 0.3^{*}$ \\
\hline
\end{tabular}

Fig. (3). Summary table of the results obtained (upper) using $1 \mathrm{~Hz}$ TMs and (lower) $15 \mathrm{~Hz}$ TMS. The values are the mean \pm SEM for a total of 40 repetitions in the 4 animals. Significant differences $(\mathrm{P}<0.05)$ are marked by *. The cartoon (top) illustrates the relative position of the skull-mounted electrodes $(\mathrm{Tl}=$ left temporal, $\operatorname{Tr}=$ right temporal, etc.) *marks the location of the centre of the TMS coil.

We have sampled 11 cells under these conditions. Overall, we observed a significant delay in reaching peak time in $10 / 11$ cells (from $48 \pm 2$ to $51 \pm 2.5 \mathrm{~ms}$ ), with an overall reduction of cell firing of $19 \pm 9 \%$ and a reduction in the duration of the response measured at half-height (from $28 \pm 3.1$ to $24.1 \pm 3.3 \mathrm{~ms})$.

\section{DISCUSSION}

Enhancement of delta power in the EEG as a result of low frequency TMS is already known [10,11]. Here we demonstrate that stimulation at higher frequencies results in an opposite effect, which is robust and reproducible. We have used the simplest available EEG setup to test the hy- 
pothesis that higher frequency stimulation could reproducibly alter the EEG in a manner consistent with the known effects already demonstrated using low frequency stimulation. The utility of this information is to provide a quick and easy way to predict likely effects of rTMS protocols, which may be complex and include both high and low frequency components [9]. This becomes more useful when TMS is used on brain regions which have no easily measurable output, such as frontal or parietal cortices. The EEG effects we have shown are strong and reproducible, and can be (re)tested over a very short interval of 60 s or less.

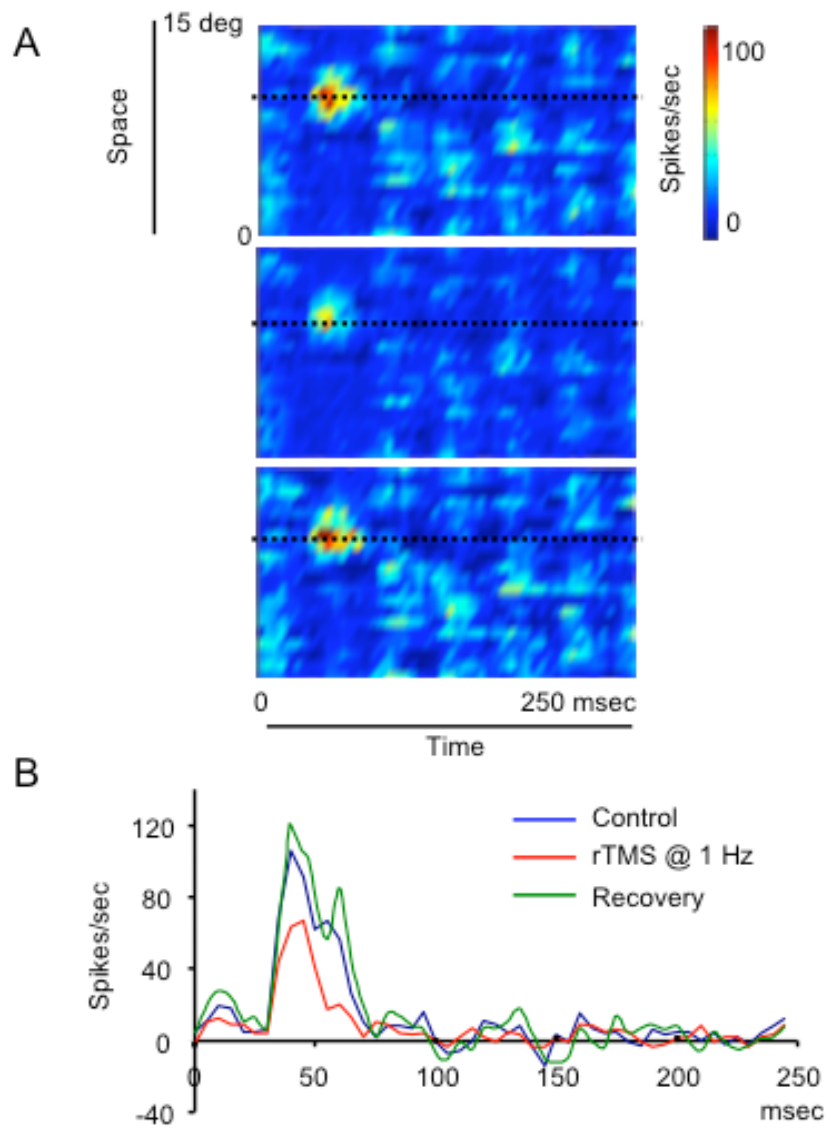

Fig. (4). A Plots showing a measure of receptive field size and responsiveness of a single cell in the visual cortex before, just after and fully recovered from $1 \mathrm{~Hz}$ TMS stimulation. Peak firing was clearly reduced, as was the effective area over which stimulation could elicit a response. B Temporal response curves obtained by slicing through the spatiotemporal profile at the center of the receptive field (indicated by the horizontal dotted line) where the maximum response was obtained. Each point represents the sum of the On and Off profiles represented by Light and Dark bar presentation [13].

The widely accepted view that the EEG is spatially nonselective has begun to be challenged [16-19]. However, the technicalities required to improve the spatial acuity of EEG are complicated and expensive. In using a simple form, as we have here, we have shown some evidence of spatial selectivity, in that increases in theta, alpha and beta wave amplitudes were seen only on recordings from the occipital and frontal electrodes and were not seen from either temporal electrode. While this may suggest some differences in the anatomical interconnections of the cortex, it may also simple be due to the relative amplitudes of the various components of the EEG. Thus, the major changes resulting from rTMS of either frequency was in the most potent, delta band, and this was seen wherever an electrode was placed, So, while the tool may be useful to predict the nature of the rTMS effect, it is unlikely to be useful in measuring the extent of such action. However, others [20] have shown that TMS can induce transient synchronization of spontaneous activity of cortical neurons within the $15-30 \mathrm{~Hz}$ frequency range when measured with simultaneous EEG. This required sophisticated recording apparatus and analysis of the EEG.

In trying to understand the mechanisms underlying the effectiveness of rTMS we have also demonstrated effects at the level of the single cell, which are commensurate with effects we previously showed giving rise to changes in the responses properties of cells located as "targets" of the cells directly affected. Put simply, suppression of the cortical area under TMS may not suppress all cells but those that are not completely blocked exhibit slow response dynamics. Indeed, while we recently showed that suppressive effects on the primary visual cortex resulted in a shift of firing from tonic to burst mode in the distant target area of thalamus the LGN [9]; this effect would be predicted on the grounds of a loss or substantive shift in the dynamics of the cortical influence. The underlying dilemma of the dynamics of the TMS situation is illustrated by the finding of others [21] who have shown that a single pulse of TMS at stimulation intensities higher than $50 \%$ of the stimulator output can create a $200 \mathrm{~ms}$ period of depressed activity in the visual cortex followed for a transient rebound (up to $500 \mathrm{msec}$ ) and a later depression. In our case, continuous rTMS at $1 \mathrm{~Hz}$ appeared to induce an effect which was observed after the stimulation and lasted for several minutes, indicating a simple, prolonged depression of cortical activity at the core of the TMS region of "effect", vide supra and $[9,10]$.

In using TMS as a tool to either "excite" or "inhibit" cortical function, it is also important to remember that measurable effects of TMS such as those we now demonstrate on the EEG may contain elements deriving from the "knock-on" effect directly attributable to circuit connections within cortex (both near and remote) and also the underlying connections to deeper structures, acting transynaptically, via withdrawal of excitatory influences and subsequent changes in the local excitatory/inhibitory balance.

\section{CONCLUSIONS}

A simple measure of EEG activity can be useful as a tool to infer the polarity of a TMS induced cortical effect, and may be developed to indicate degree of spread of activity, bearing in mind that effects of TMS may be both local and distant.

\section{ACKNOWLEDGEMENTS}

This study was supported by MEC (BFU2005-00502) and Xunta de Galicia (PGIDIT06PXIB137036PR and Consellería de Educación-2007/000140-0), Spain.

\section{REFERENCES}

[1] Wassermann EM, Lisanby SH. Therapeutic application of repetitive transcranial magnetic stimulation: a review. Clin Neurophysiol $2001 ; 112: 1367-77$. 
[2] Ridding MC, Rothwell JC. Therapeutic use of rTMS. Nat Rev Neurosci 2007 ; (Epub ahead of print).

[3] Pascual-Leone A, Valls-Sole J, Wassermann EM, Hallett M. Responses to rapid-rate transcranial magnetic stimulation of the human motor cortex. Brain 1994; (Pt 4):847-58.

[4] Wassermann EM, Grafman J, Berry C, et al. Use and safety of a new repetitive transcranial magnetic stimulator. Electroencephalogr Clin Neurophysiol 101: 412-7.

[5] Chen R, Classen J, Gerloff C, et al. Depression of motor cortex excitability by low frequency transcranial magnetic stimulation. Neurology 48: 1398-403.

[6] Pascual-Leone A, Houser CM, Reese K, et al. Safety of rapid-rate transcranial magnetic stimulation in normal volunteers. Electroencephalogr Clin Neurophysiol 1993; 89(2): 120-30.

[7] Berardelli A, Inghilleri M, Rothwell JC, et al. Facilitation of muscle evoked responses after repetitive cortical stimulation in man. Exp Brain Res 1998; 122: 79-84.

[8] Kujirai T, Caramia MD, Rothwell JC, et al. Corticocortical inhibition in human motor cortex. J Physiol 1993; 471: 501-19.

[9] de Labra C, Rivadulla C, Grieve K, Marino J, Espinosa N, Cudeiro J. Changes in visual responses in the feline dLGN: selective thalamic suppression induced by transcranial magnetic stimulation of V1. Cereb Cortex 2007;17:1376-85.

[10] Rivadulla C, De Labra C, Fernández Del Olmo M, Cudeiro J. Effects of repetitive transcranial magnetic stimulation (rTMS) evaluated by EEG recording in the anaesthetized cat. FENS Abstr 2004; 2: A122.11.

[11] Aydin-Abidin S, Moliadze V, Eysel UT, Funke K. Effects of repetitive TMS on visually evoked potentials and EEG in the anaesthetized cat: dependence on stimulus frequency and train duration. J Physiol 2006; 574(Pt 2): 443-55.

[12] Rivadulla C, Martinez LM, Varela C, Cudeiro J. Completing the corticofugal loop: a visual role for the corticogeniculate type 1 metabotropic glutamate receptor. J Neurosci 2002; 22: 2956-62.
[13] Cai D, DeAngelis GC, Freeman RD. Spatiotemporal receptive field organization in the lateral geniculate nucleus of cats and kittens. J Neurophysiol 1997; $78:$ 1045-61.

[14] DeAngelis GC, Ohzawa I, Freeman RD. Spatiotemporal organization of simple-cell receptive fields in the cat's striate cortex. I. General characteristics and postnatal development. J Neurophysiol 1993; 69: 1091-117.

[15] Jones JP, Palmer LA. The two-dimensional spatial structure of simple receptive fields in cat striate cortex. J Neurophysiol 1987; 58: 1187-211.

[16] Babiloni F, Babiloni C, Locche L, Cincotti F, Rossini PM, Carducci F. High-resolution electro-encephalogram: source estimates of Laplacian-transformed somatosensory-evoked potentials using a realistic subject head model constructed from magnetic resonance images. Med Biol Eng Comput 2000; 38: 512-9.

[17] Babiloni F, Cincotti F, Mattiocco M, et al. Brain computer interface: estimation of cortical activity from non invasive high resolution EEG recordings. Conf Proc IEEE Eng Med Biol Soc 2004; 6: 4375-6.

[18] Babiloni C, Babiloni F, Carducci F, et al. Human cortical responses during one-bit short-term memory. A high-resolution EEG study on delayed choice reaction time tasks. Clin Neurophysiol 2004; 115 161-70.

[19] Huber R, Esser SK, Ferrarelli F, Massimini M, Peterson MJ, Tononi G. TMS-induced cortical potentiation during wakefulness locally increases slow wave activity during sleep. PLoS ONE 2007; 2(3): e276.

[20] Paus T, Sipila PK, Strafella AP. Synchronization of neuronal activity in the human primary motor cortex by transcranial magnetic stimulation: an EEG study. J Neurophysiol 2001; 86: 1983-90.

[21] Moliadze V, Zhao Y, Eysel U, Funke KD. Effect of transcranial magnetic stimulation on single-unit activity in the cat primary visual cortex. J Physiol 2003; 553(Pt 2): 665-79. 